\section{Umweltdaten generalüberholt}

\author{
Umweltbundesamt (Hg.): Daten zur \\ Umwelt 2005. Der Zustand der Umwelt \\ in Deutschland. Erich-Schmidt Verlag, \\ 2006, 350 S., plus CD-ROM, ISBN 3-503- \\ 09057-6, Euro 46,80; auch komplett als \\ CD-ROM erhältlich: ISBN 3-503-09058-4, \\ Euro 36,80
}

\section{Rezension von Gerhard Sardemann, ITAS}

\section{Entwicklung seit 1984}

Bei den „Daten zur Umwelt“ handelt es sich um eine ressort- und themenübergreifende Darstellung der in Deutschland erhobenen Umweltdaten mit langer Tradition. Die Ausgabe 2005 ist die achte seit dem ersten Erscheinen im Jahr 1984. Damals bekam der vom Waldsterben aufgeschreckte und durch ,ferntransportierten" Smog aus dem Osten geplagte Bundesbürger (West) eine Art Atlas an die Hand, in dem er mit dem Finger auf großformatigen Abbildungen, vor allem Karten, seine Eindrücke nachvollziehen konnte. Erklärender Text war auf ein Mindestmaß beschränkt: Drei Sätze auf der einen DIN A4 Seite umrissen den Sachverhalt, alles andere zeigte das Bild auf der gegenüberliegenden Seite. Diese Lücken wurden in den nachfolgenden Auflagen immer mehr mit Text und Tabellen gefüllt, die anfänglich schlechte Druckqualität verbessert und die Karten, nun aufklappbar, immer größer und auch unhandlicher. Auch das Volumen wuchs von ehemals 400 Seiten auf über 600 Seiten an.

In der Ausgabe der ,Daten zur Umwelt 1990/91“, der vierten nach 1984, wurde erstmals der Umweltzustand in Deutschland zum Zeitpunkt der Wiedervereinigung dargestellt schlaglichtartig und getrennt für alte und neue Bundesländer. Den nachfolgenden Ausgaben konnte man dann entnehmen, dass sich die Bedingungen in den alten und neuen Ländern langsam anglichen. Der Schadstoffausstoß in den neuen Ländern verminderte sich stark durch den Bau von Filtern und Kläranlagen, insbesondere aber auch durch Betriebsschließungen bzw. -modernisierungen, während durch eine Zunahme des Individualverkehrs und des Hausmüllaufkommens neue Probleme sichtbar wurden.
Nach der Konferenz der Vereinten Nationen über Umwelt und Entwicklung im Jahre 1992 in Rio de Janeiro ging es in den ,Daten zur Umwelt 1992/93“ zum ersten Mal auch um globale Umweltprobleme. Angesprochen wurden der Abbau der Ozonschicht, der Treibhauseffekt und die Bestandsentwicklung der tropischen Wälder. Dabei handelt es sich durchweg um Phänomene, die der direkten Erfahrung eines Mitteleuropäers nicht unbedingt zugänglich sind und einen von vornherein größeren Erklärungsbedarf haben, als die meisten Umweltprobleme im eigenen Land. Diesem Erklärungsbedarf wurde zwar erst in späteren Auflagen der „Daten zur Umwelt“" Rechnung getragen; er ist aber vielleicht auch ein Grund dafür, dass aus dem ursprünglichen „Umweltatlas“ ein recht textlastiges ,Umweltkompendium“" wurde.

Ein weiterer Grund für diese Entwicklung kündigte sich mit dem ersten Erscheinen der „Daten zur Umwelt“" auf CD-ROM im Jahr 1997 an. Mit dem gleichzeitigen Ausbau der Präsenz des Umweltbundesamtes im Internet und den dort zur Verfügung gestellten Umweltdaten erhalten die „Daten zur Umwelt“" zudem eine Konkurrenz, die viele zunächst davon abgehalten haben könnte, sich eine gedruckte Ausgabe anzuschaffen. Sicher gilt dies für einzelne Grafiken und Tabellen, für deren Darstellung man den Bildschirm in der Wissenschaft schon wesentlich länger nutzt, als es das World Wide Web gibt. Ganz anders sieht es dagegen aus, wenn es um eine zusammenhängende Darstellung von Umweltproblemen mit viel beschreibendem Text geht. Hier ist die gedruckte Darstellung derjenigen auf dem Bildschirm um einiges voraus - insbesondere dann, wenn beide Darstellungsarten miteinander kombiniert werden, wie es bei der aktuellen Ausgabe der „Daten zur Umwelt" der Fall ist: Zusätzlich zum Text (gedruckt oder als pdf-Datei) werden Grafiken und Tabellen auf CD-ROM mitgeliefert. Die pdf-Datei kann man nach Schlagworten durchsuchen, die zusätzlichen Darstellungen sind hilfreiche Ergänzungen des im Text schon enthaltenen Datenmaterials.

Bei der neuesten Ausgabe 2005 der „Daten zur Umwelt" hat man durch Auslagerung zahlreicher Daten auf CD-ROM und ein wesentlich feineres Layout inzwischen ein handliches Volumen von 352 Seiten erreicht. Diese stark gesteigerte Qualität schlägt sich auch im 
Preis nieder, der von anfänglichen $15 \mathrm{DM}$ inzwischen auf $46,80 €$ angestiegen ist.

\section{Neuer thematischer Aufbau}

Als Neuerung der hier zu besprechenden Ausgabe fällt die darin zum ersten Mal eingeführte, am jeweils ,zu schützenden Gut“ orientierte Gliederung sofort ins Auge. Sie umfasst die folgenden Bereiche:

- Klima,

- Umwelt, Gesundheit und Lebensqualität (mit den Unterkapiteln „Unerwünschte Stoffe und Schadstoffe“, „Lärm“ und „Strahlen“),

- Umweltmedien und Ökosysteme (mit den Unterkapiteln „Luftreinhaltung“, „Gewässerschutz“, „Bodenschutz“, „Ökosysteme und biologische Vielfalt"),

- Nachhaltige Nutzung natürlicher Ressourcen und Bewirtschaftung von Abfällen.

Der Herausgeber folgt hier einer durch das sechste Europäische Umweltaktionsprogramm vorgegebenen Einteilung und strebt eine integrierte Betrachtung von Umweltaspekten zusammen mit wirtschaftlichen und querschnittsorientierten Aktivitäten an. Dabei geht es insbesondere darum, ,die politischen Anstrengungen für eine nachhaltige Entwicklung unserer Lebensgrundlagen besser abzubilden" (S. 5). Die früher verwendete Unterteilung in die Bereiche „Natur und Landschaft", „Wald“, „Boden“, „Luft“, „Wasser“, „Meere“, „Abfall“, „Lärm“, „Umwelt und Gesundheit" sowie „Strahlenschutz" schaut allerdings an einigen Stellen immer noch recht deutlich hinter der neuen Einteilung hervor: Insbesondere verwirrt, dass im fortlaufenden Text die Kapitelüberschriften ganz weggelassen wurden und allein an der Farbgebung der Kopfzeile zu erkennen ist, in welchem übergeordneten Kapitel man sich gerade befindet. Was die wirtschaftlichen und querschnittsorientierten Daten angeht, so hatte man früher dafür ein eigenes Kapitel „Allgemeine Daten“, später „umweltrelevante Aktivitäten“. Die ehemals darin enthaltenen Informationen finden sich nun verstreut im Text; ein ganz besonders großer Teil davon, insbesondere derjenige über die wirtschaftliche Entwicklung und den Energieverbrauch, ist im Kapitel „Klima“ zu finden.

\section{Themenbereich „Klima“}

Bei diesem Bereich ist die integrierte Darstellung eines „Umweltproblems“ mit seiner Erkennung, seinen Ursachen, seinen möglichen Auswirkungen und den erforderlichen sowie den bereits ergriffenen Maßnahmen (und deren Rückwirkungen auf Wirtschaft und Gesellschaft wie z. B. bei der ökologischen Steuerreform) sicherlich am weitesten vorangeschritten. Es geht darin um eine Beschreibung des heutigen Klimas, der Funktion und Wirkung von Treibhausgasen, deren Aufkommen bei nahezu allen menschlichen Aktivitäten und wie man anthropogene Emissionen von Treibhausgasen im nationalen und internationalen Rahmen reduzieren möchte. Kompilierungen dieser Art fand man bislang in den regelmäßig erscheinenden „Nationalberichten Deutschlands zur Klimarahmenkonvention", deren letzter allerdings schon im Jahre 2002 erschien. Hier hat man also einen etwas aktuelleren Überblick (die Zahlen enden in der Regel im Jahr 2004), außerdem fließen über die im Kioto-Protokoll zur Klimarahmenkonvention hinausgehende Zielvorstellungen mit ein, welche im „Fortschrittsbericht zur nationalen Nachhaltigkeitsstrategie 2004“ und im „Nationalen Klimaschutzprogramm 2005“ für den Zeitraum bis zum Jahr 2020 ihren Niederschlag fanden (S. 28).

Als Meteorologe fragt man sich allerdings manchmal, was das Klima als sog. ,Schutzgut" anderen komplexen Systemen voraushat, dass man es derart exponiert in einem solchen Bericht behandeln müsste. Die Einteilung scheint also recht deutlich dem aktuellen Stellenwert auf der politischen Agenda geschuldet zu sein. Vor etwas mehr als zehn Jahren, als das Thema zum ersten mal in den „Daten zur Umwelt“ auftauchte, hätte womöglich auch der Abbau der Ozonschicht zusammen mit den nötigen Maßnahmen zu dessen Eindämmung ein eigenes Kapitel bekommen. Das Thema findet sich nun aber als Unterpunkt des Bereichs „Umwelt, Gesundheit und Lebensqualität“, wo im Kapitel „Strahlen“ auf die solare UV-Strahlung und den Abbau der stratosphärischen Ozonschicht eingegangen wird - insbesondere auf die „Exposition der Bevölkerung gegenüber nichtionisierender Strahlung“. 


\section{Themenbereich „Umweltmedien und Ökosysteme"}

Auf den Zustand der tropischen Wälder wird in den vorliegenden „Daten zur Umwelt“ gar nicht mehr eingegangen. Im Bereich „Umweltmedien und Ökosysteme", mit dem Unterkapitel „Ökosysteme und biologische Vielfalt", geht der Blick über die europäischen Wälder nicht hinaus; auch der Einfluss internationaler Abkommen und Standards wird nur für den deutschen Wald diskutiert. Der Rezensent könnte sich sehr gut vorstellen, dass zumindest zusätzlich zu der bestehenden Angabe zertifizierter Waldflächen in Deutschland (ein Maß für die Nachhaltigkeit der Waldbewirtschaftung, S. 269), auch der deutsche Verbrauch von Holz aus zertifizierten und nicht zertifizierten Waldflächen weltweit dargestellt wird.

\section{Themenbereich „Nachhaltige Nutzung natürlicher Ressourcen und Bewirtschaf- tung von Abfällen“}

Dieser schnelle, zunächst nur an der Einordnung globaler Umweltprobleme orientierte Überflug über die ersten drei Bereiche der aktuellen ,Daten zur Umwelt" zeigt schon, dass die neu eingeführte, am jeweils ,zu schützenden Gut“ orientierte Einteilung auch nicht ohne Tücken ist. Das kann auch anhand des letzten Bereichs „Nachhaltige Nutzung natürlicher Ressourcen und Bewirtschaftung von Abfällen" exemplarisch gezeigt werden. Dort geht es unter anderem auch um den Umgang mit der Ressource „Fläche“. Hier muss man aber, um ein vollständiges Bild über deren Entwicklung zu erhalten (insbesondere über die tägliche Zunahme der Siedlungs- und Verkehrsfläche) ins Unterkapitel „Bodenschutz" zurückblättern. Hier ist sicher noch einige redaktionelle Arbeit nötig, auch um das Kapitel „Ressourcen“, das in den vorherigen Ausgaben noch gar nicht vorkam, weiter aufzuwerten. Im Falle von Wasser als Ressource ist die Trennung vom Unterkapitel „Gewässerschutz", wo es nur um die Eigenschaften und die Verschmutzung von Grund- und Oberflächenwasser geht, schon befriedigend gelöst. Andererseits fallen unerwartete Datenlücken auf, die die gesamte Wasserentnahme eines Jahres aus der Natur betreffen. Für diese zur Berechnung der Wasserproduktivität benötigte Größe fehlen in der Wasserstatistik beispielsweise Angaben zum Verbrauch in der Landwirtschaft. Der Differenzbetrag liegt in der Größenordnung des derzeitigen Verbrauchs des produzierenden Gewerbes und des Bergbaus. Während sich dieser Verbrauch, ebenso wie der des größten Verbrauchers, der Wärmekraftwerke, seit 1991 stark vermindert hat, hat ausgerechnet der in der Statistik nicht ausgewiesene Anteil (insbesondere wohl derjenige in der Landwirtschaft) eine eher ansteigende Tendenz. Hier besteht aus Sicht des Rezensenten Erklärungsbedarf, der im vorliegenden Band nicht befriedigt wird.

\section{Potenzial für weitere Verbesserungen}

Auf alle in den „Daten zur Umwelt“ abgedeckten Themenbereiche soll und kann in dieser Rezension nicht eingegangen werden. Beim Vergleich der aktuellen Ausgabe mit vorangegangenen Auflagen kam beim Rezensenten allerdings der Wunsch auf, etwas über die Entwicklung des Datenbestandes zu erfahren: Was hat sich an der Datenlage verbessert, wo sind altbekannte Lücken noch immer nicht geschlossen worden, wo gibt es Brüche in den Statistiken und wo besteht aufgrund neuer Erkenntnisse oder erweiterter Fragestellungen Bedarf an neu zu erhebenden oder zusätzlich in die Darstellung in den „Daten zur Umwelt" aufzunehmenden Größen und Indikatoren? Was letztere betrifft, so gibt es zusätzlich das Umwelt-Kernindikatorensystem, dessen Gliederung grundsätzlich mit der nun in den „Daten zur Umwelt" verwendeten übereinstimmt. Es gibt zudem das Umwelt-Barometer und den Deutschen Umweltindex. Diese Systeme weiter zu integrieren, dürfte eine viel versprechende Aufgabe für die Zukunft darstellen. Was die Ausgabe 2005 angeht, so ist sicher schon ein Schritt auf diesem Weg gemacht worden. Der Vergleich von Umwelttrends mit quantifizierten Umweltzielen, wie sie beispielsweise in der deutschen Nachhaltigkeitsstrategie genannt werden, ist jedoch in der vorliegenden Ausgabe noch zu wenig systematisch durchgeführt worden. In manchen Kapiteln wurde auf Umweltziele eingegangen, in anderen nicht. 


\section{Fazit}

Experten werden in ihrem Fachgebiet sicherlich Lücken finden, die dadurch entstanden, dass die jeweiligen Daten nicht erhoben sind oder die Redaktion sie in der vorliegenden Form nicht für veröffentlichenswert hielt. Lästig sind offensichtliche Druckfehler, insbesondere vertauschte Abbildungen. Das geht aber über ein zu erwartendes Maß nicht hinaus und kann bei nachgereichten Auflagen sicherlich behoben werden. Der Zugriff auf die Daten war in den früheren Auflagen in etwas direkterer Form möglich; der PC ermöglicht allerdings heute, im Text versteckte Daten schnell aufzufinden sowie zusätzliche Datenquellen im Internet zu erschließen. Das Aufzeigen von Zusammenhängen im Text kann durchaus als Verbesserung der aktuellen Ausgabe der „Daten zur Umwelt“ gegenüber früheren angesehen werden. Es wird allerdings deutlich, dass die neu eingeführte, am jeweils „zu schützenden Gut“ orientierte Einteilung nicht ohne Tücken ist. Insbesondere wurde der Anspruch, eine integrierte Darstellung der Umwelt zu liefern, zusammen mit einer Überprüfung von Maßnahmen zu ihrer Erhaltung im Rahmen einer nachhaltigen Entwicklung, noch nicht in allen Punkten erfüllt. Besonders positiv hat der Rezensent allerdings die Tatsache empfunden, nach längerer Zeit wieder einmal realiter in einem aktuellen Exemplar blättern zu dürfen; von der Haptik her hat das dem „browsen“ in online-Exemplaren oder elektronischen Datenbanken einiges voraus.

》)

\section{Wenn Wissenschaftler Staub sammeln}

J. Soentgen, K. Völzke (Hg.): Staub Spiegel der Umwelt. Stoffgeschichten Band 1. München: oekom Verlag, 2005, 272 S., ISBN 3-936581-60-6, Euro 29,80

\section{Rezension von Norbert Nowotsch, Fach- hochschule Münster}

Pünktlich zum Erscheinen dieses Buches waren die Zeitungen voll „staubiger Leitartikel“. Eine bessere - und dazu kostenlose - PR-Unterstüt- zung hätte es sich nicht wünschen können. Zum einen - fast ein Dauerbrenner - war es das unangenehme Thema „Feinstaubbelastung“ in den Metropolen der Republik, zum anderen das Wunder des kosmischen Staubes, von dem einige Körnchen auch in Deutschland erforscht werden durften. ${ }^{1}$

Laut Klappentext ist dieser Band mit einem Überblick über die aktuelle Staubforschung der Auftakt zu einer Reihe mit dem Titel „Stoffgeschichten" - und das nicht ohne Grund, wie gleich zu Anfang programmatisch vermittelt wird: "Denn der Staub ist so etwas wie der Anfang alles Stofflichen - und sein Ende."

Jens Soentgen, der wissenschaftliche Leiter des Wissenschaftszentrums Umwelt der Universität Augsburg, nimmt als Herausgeber diese Vorgabe auf und entwickelt im ersten Beitrag des Bandes auf kompakte und unterhaltsame Weise eine kleine „Kulturgeschichte des Staubes". Diese ist nicht nur eine Einführung in das Thema, sondern auch eine inspirierte Anregung zum Weiterlesen und darüber hinaus eine Ermutigung zum Stöbern in weiteren Werken dieses Themenfeldes. Die folgenden sechzehn Beiträge spannen den thematischen Bogen über viele, wenn auch vornehmlich naturwissenschaftlich orientierte Bereiche; fast alle Autoren halten sich aber trotz Faktenfülle an einen angenehm flüssigen und unterhaltsamen Sprachstil.

Auch der Erforscher des eingangs erwähnten Sternenstaubes ist mit einem Beitrag im Buch vertreten. Noch in Vorfreude auf die "Stardust Memories” (zum Zeitpunkt der Abfassung seines Textes war die Sammelaktion der NASA Raumsonde noch nicht erfolgreich abgeschlossen) erinnert Thomas Stephan unter diesem schönen Titel an die Entstehungsgeschichte unseres Sonnensystems aus einer riesigen Gas- und Staubwolke und vermittelt die Bedeutung der Mission Stardust zur Erforschung dieses Prozesses und damit natürlich unserer eigenen Herkunft.

Einen Ausflug in die Welt des ganz Kleinen unternimmt Manfred Euler in seinem Beitrag ,Tanzende Staubkörner und Nanomaschinen"; letztere nennt er sehr schön ,intelligenten Staub". Zum klassischen Staub findet sich hier eine Abbildung der so genannten Brown'schen Molekularbewegung eines Staubteilchens, von Euler „Zittern des Staubes” genannt. Man denkt intuitiv an die zahlreichen lyrischen Um- 